\title{
L'analyse par cytométrie de masse des muscles âgés et dystrophiques permet d'identifier des nouvelles populations distinctes des cellules satellites
}

\author{
Lorenzo Giordani, Gary J. He, Elisa Negroni, Hiroshi Sakai, Justin Y.C. Law, M. Mona Siu, \\ Raymond Wan, Shahragim Tajbakhsh, Tom H. Cheung, Fabien Le Grand
}

Lorenzo Giordani Elisa Negroni Fabien Le Grand Sorbonne Université, Inserm UMRS974, Association Institut de Myologie, Centre de Recherche

en Myologie, 75013 Paris,

France

Gary J. He

Justin Y.C. Law

M. Mona Siu

Raymond Wan

Tom H. Cheung

Division of Life Science,

Center for Stem Cell

Research, State Key

Laboratory of Molecular

Neuroscience, Center

of Systems Biology and

Human Health, Institute

for Advanced Study,

The Hong Kong

University of Science

and Technology, Clear

Water Bay, Kowloon,

Hong Kong, China

Hiroshi Sakai

Shahragim Tajbakhsh

Stem Cells and

Development,

Department

of Developmental and

Stem Cell Biology, Institut

Pasteur, 75015 Paris,

France

CNRS UMR 3738, Institut

Pasteur, 75015 Paris,

France

Contact

lmgiordani@gmail.com
Le muscle squelettique adulte est une structure complexe dotée d'un potentiel de régénération remarquable. Cette capacité repose sur l'interaction orchestrée entre différentes populations musculaires qui résident dans le tissu [1]. Au cours du processus de vieillissement naturel ou dans des conditions de maladies chroniques, telles que la dystrophie musculaire, des changements spécifiques se produisent dans une ou plusieurs de ces populations, entraînant une perte progressive de la capacité de régénération. Ceci conduit in fine à la sarcopénie, la fibrose, l'infiltration graisseuse et, dans des conditions pathologiques à la nécrose $[2,3]$.

Malgré d'importants travaux de recherche conduits dans ce domaine, nous sommes loin de comprendre complètement ces changements. Même lorsque la cause génétique est connue, comme par exemple dans la dystrophie musculaire de Duchenne (DMD), nous sommes toujours incapables de préciser toutes les étapes qui mènent du défaut moléculaire jusqu'à l'expression de la maladie.

La raison principale de cette ignorance provient du manque d'outils permettant d'appréhender l'hétérogénéité de ces populations cellulaires. Les méthodes conventionnelles, capables seulement de fournir des informations moyennées, ont ralenti nos tentatives destinées à caractériser les changements pathologiques du microenvironnement, laissant ces souspopulations dysfonctionnelles cachées. D'où la nécessité de disséquer l'hétérogénéité du muscle au niveau de cellules isolées.

Notre projet est donc d'identifier les changements au niveau cellulaire qui interfèrent avec la bonne exécution du programme de régénération au cours du vieillissement, et en condition pathologique, en utilisant la spectrométrie de masse (technologie CyTOF). Des travaux de recherche récents suggèrent que la perte du potentiel régénératif du muscle pendant le vieillissement et en pathologie de type dystrophique est un processus multifactoriel dans lequel l'hétérogénéité cellulaire joue un rôle central. Il est frappant que cet épuisement fonctionnel progressif ne soit pas un processus dichotomique. Il s'agit plutôt d'une "dérive " progressive vers l'état pathologique lequel génère un gradient de sousfractions semi-fonctionnelles intermédiaires au sein des populations résidentes du muscle. Les régulateurs moléculaires qui contrôlent la transition entre ces étapes sont la clé pour comprendre et contrer la progression du défaut. Cependant, lorsque les populations musculaires sont étudiées avec des méthodes classiques, ces sous-fractions restent indétectables. Afin de caractériser ces interrupteurs moléculaires, nous avons besoin d'un outil pour détecter de façon non biaisée les différentes sousfractions et les étudier comme des entités discrètes. Cela ne peut être réalisé qu'en profilant le muscle entier jusqu'à la résolution d'une seule cellule. Étonnamment, à ce jour, il n'y a pas d'études publiées qui traitent de la complexité phénotypique du muscle squelettique au niveau unicellulaire dans la dystrophie musculaire de Duchenne ou lors du vieillissement physiologique.

Profitant de la technologie CyTOF [4], nous avons profilé, à une échelle unicellulaire, les différentes populations au cours du vieillissement musculaire et en pathologie (en utilisant des jeunes et vieux $\mathrm{mdx} 4 \mathrm{cv}$ - un modèle de DMD). Ceci nous a permis de définir, en comparaison avec le jeune muscle sain, un schéma de ces états biologiques révélant des similitudes fonctionnelles et mettant en évidence des motifs récurrents. En utilisant une approche basée sur l'application de différents algorithmes de visualisation et de groupement tels que SPADE, viSNE [5] et Statistical Scaffold, nous avons pu séparer et comparer l'hétérogénéité des différentes conditions à une profondeur sans précédent. Nous avons pu identifier des sous-ensembles cellulaires précédemment négligés que nous avons isolés et caractérisés in vitro. Les caractéristiques particu- 


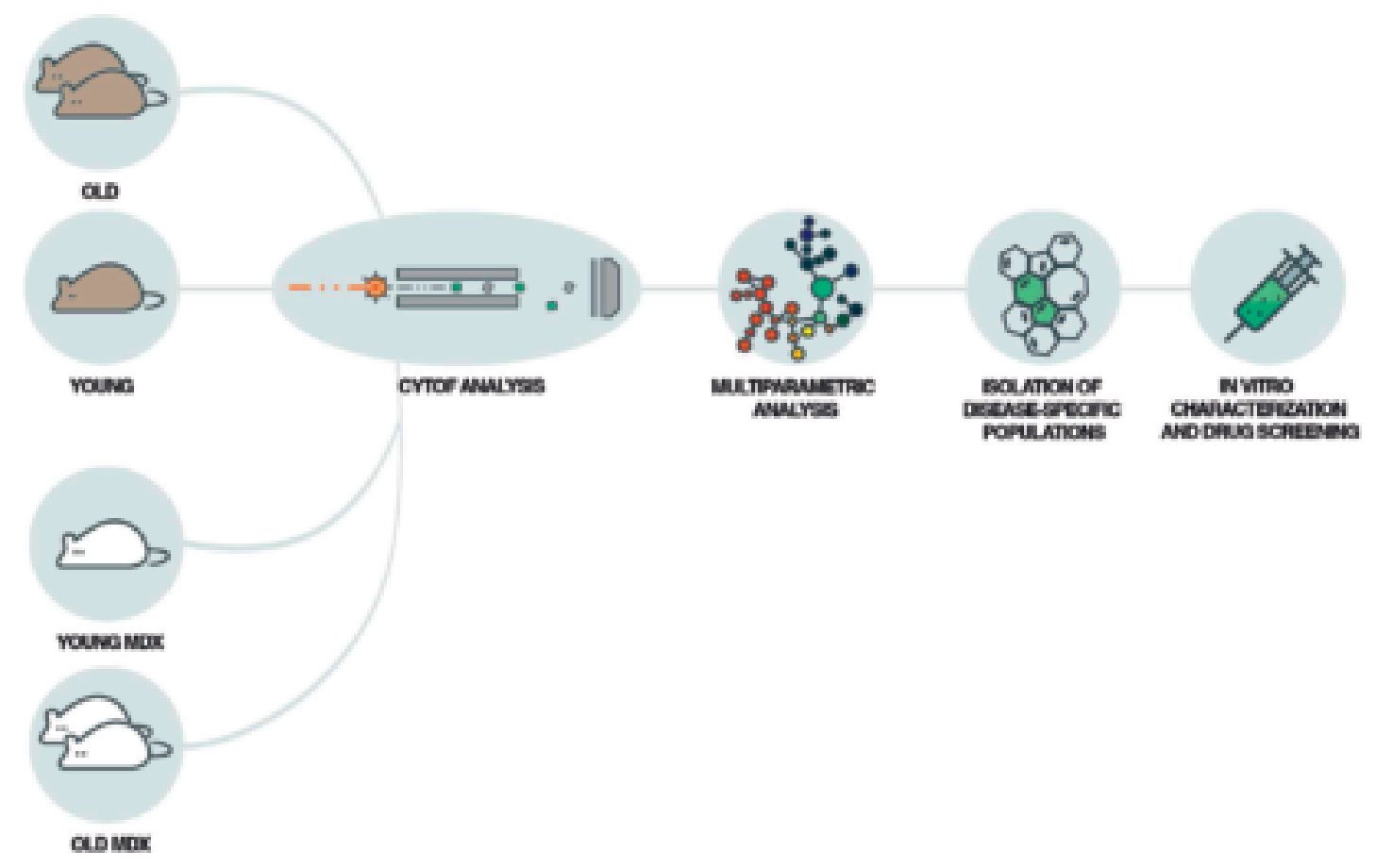

Figure 1

Schéma du workflow développé pour identifier les sous-populations maladie-spécifiques.

lières de ces populations plaident fortement en faveur de leur implication dans la régénération musculaire. Pour cette raison, nous étudions maintenant les changements dynamiques qui se produisent dans ces cellules chez les souris $\mathrm{mdx} 4 \mathrm{c}$ jeunes et âgées. Il s'agit de la première tentative destinée à étudier simultanément la totalité de la fraction mononucléée du muscle sain et dystrophique, et à suivre son évolution au cours du vieillissement à une résolution de cellule unique.

La caractérisation des différentes populations cellulaires présentes dans le muscle fournira de nouvelles informations sur le processus de régénération, élargissant davantage notre compréhension de la façon dont les cellules individuelles coordonnent les réponses différentielles pour parvenir à la réparation du muscle squelettique après une blessure. En outre, l'étude de sous-populations spécifiques associées à une maladie fournira non seulement une base solide pour l'identification future de biomarqueurs diagnostiques, mais mettra également en évidence d'éventuelles cibles pharmaceutiques, ouvrant ainsi la voie à de nouvelles thérapies pour les maladies myodégénératives.

Nous prévoyons que, dans un proche avenir, des technologies telles que la transcriptomique et la protéomique unicellulaires seront combinées avec la médecine personnalisée pour fournir un traitement adapté à chaque patient. Notre ensemble de données, aux côtés de nombreuses autres données qui visent à profiler les cellules de différents modèles de maladies, servira d'atlas de référence pour naviguer dans la grande quantité de données qui seront inévitablement générées par une telle approche combinée.

Single-cell mass cytometry analysis of aged and dystrophic muscle identifies novel populations distinct from satellite cells

\section{LIENS D'INTÉRÊT}

Les auteurs déclarent n'avoir aucun lien d'intérêt concernant les données publiées dans cet article.

\section{RÉFÉRENCES}

1. Bentzinger CF, Wang YX, Rudnicki MA. Building muscle: molecular regulation of myogenesis. Cold Spring Harb Perspect Biol 2012 ; 4. pii : a008342. doi : 10.1101/cshperspect.a008342.

2. Kharraz Y, Guerra, J, Pessina P, et al. Understanding the process of fibrosis in Duchenne muscular dystrophy. Biomed Res Int $2014 ; 965631$.

3. Brack AS, Rando TA. Intrinsic changes and extrinsic influences of myogenic stem cell function during aging. Stem Cell Rev 2007 ; 3: 226-37.

4. Janes MR, Rommel C. Next-generation flow cytometry. Nat Biotechnol 2011; 29 : 602-4.

5. Diggins KE, Ferrell PB, Irish JM. Methods for discovery and characterization of cell subsets in high dimensional mass cytometry data. Methods $2015 ; 82$ : 55-63. 